\title{
Breast Carcinoma Progression and Tumour Vascular Markers Related to Apoptotic Mechanisms
}

\author{
Miroslava Bilecova-Rabajdova, ${ }^{1}$ Peter Urban, ${ }^{1}$ Kristina Gregova, ${ }^{2}$ Jan Varga, ${ }^{3}$ \\ Viera Fialkovicová, ${ }^{3}$ Peter Kruzliak, ${ }^{4}$ and Maria Marekova ${ }^{1}$ \\ ${ }^{1}$ Department of Medical and Clinical Biochemistry and LABMED, Faculty of Medicine, P. J. Šafárik University in Košice, \\ Trieda SNP 1, 04011 Košice, Slovakia \\ ${ }^{2}$ Department of Histology and Embryology, Faculty of Medicine, P. J. Šafárik University in Košice, Šrobárova 2, \\ 04011 Košice, Slovakia \\ ${ }^{3}$ 2nd Department of Gynecology and Obstetrics, Faculty of Medicine, P. J. Šafárik University in Košice, Rastislavova 43, \\ 04011 Košice, Slovakia \\ ${ }^{4}$ International Clinical Research Center, St. Anne’s University Hospital, Pekařská 53, 65691 Brno, Czech Republic
}

Correspondence should be addressed to Miroslava Bilecova-Rabajdova; miroslava.bilecova-rabajdova@upjs.sk and Peter Kruzliak; peter.kruzliak@savba.sk

Received 31 October 2013; Accepted 3 January 2014; Published 18 February 2014

Academic Editor: Ralf Lichtinghagen

Copyright ( 2014 Miroslava Bilecova-Rabajdova et al. This is an open access article distributed under the Creative Commons Attribution License, which permits unrestricted use, distribution, and reproduction in any medium, provided the original work is properly cited.

\begin{abstract}
Background. In the last few years, the cancer research had tried to identify and characterize new biochemical and molecular pathways in which the inhibition induces prosurvival mechanisms. Our work describes the expression of two different members of apoptotic regulatory pathway and their relationship with a progression of breast carcinoma. Materials and Methods. We compared expression of genes related to apoptosis (DR6 and Gpm6B) in the blood of patients suffering from stage I of breast cancer in different grades (I-IV), with healthy controls. After isolation of mRNA, transcription of mRNA into the cDNA was performed. The quantification of gene expression changes in DR6 and Gpm6B was detected by RT-PCR method. Analysis at the protein level was performed by the Western blot.Results. In statistical analysis of Dr6 mRNA level changes we detected significant increase starting in Grading 1 (G1) and reached maximal level in G3.This expression on mRNA levels was similar to protein levels, which copy rising tendency with maximal value in G3. The results of Gpm6B were significantly lower.Conclusion. This result showed that antiapoptotic signalling during neovascularization is increased significantly. It would be advisable in the future to study the influence of cytostatic treatment on the expression of genes related to apoptotic pathways and their relationship with progression of breast cancer tumours.
\end{abstract}

\section{Introduction}

Normal cell development is controlled by a balance between cell proliferation and apoptosis, and there is strong evidence that tumour growth is not only a result of uncontrolled proliferation but also a result of reduced apoptosis. The balance between proliferation and apoptosis is crucial in determining the overall growth or regression of the tumour. Cancer cells overcome cell death or apoptosis due to mutation of tumour suppressor genes and impaired apoptotic machinery executing cell death [1]. Another essential process important for the growth and metastasis of solid tumours is angiogenesis. The balance of endothelial cell (EC) proliferation and apoptosis is a major determinant in tumour angiogenesis, endothelial cell survival, and apoptosis in the tumour vasculature [2]. For malignant growth, solid cancers must stimulate the formation of new blood vessels by producing vascular endothelial growth factor (VEGF-A), which is required for the survival of tumour-associated vessels.

As a result of higher levels of VEGF-A, Seaman et al. [3] pointed out utilization of tumour-endothelial markers during carcinoma differentiation. Endothelial cell of the tumour, population of perivascular cells, and vascular leukocytes (VLC) are the producers of specific tumour vascular markers 
(TVM) [4]. To assess the level of TVM expression (DR6 and Gpm6B) and explore its potential pro- and antiapoptotic role during tumour development, we examined the expression of DR6 and Gpm6B mRNAs by RT-PCR and Western blot analysis isolated from human peripheral whole blood. These TVM were specifically chosen according to the journal paper of Buckanovich et al. [5], who declared that these markers are suitable for the detection of breast tumours. The overexpression of DR6 and Gpm6B leads to abnormal signalling pathways that contribute to the regulation of apoptotic processes in EC and tumour cells.

Our work describes the expression of two different members of apoptotic regulatory pathway and their relationship with a progression of breast carcinoma. This approach could help with the development of effective antiangiogenic therapies, which require identifying biologically relevant molecular targets associated with increased tumour angiogenesis [6].

The first TVM with antiapoptotic activity whose expression was examined is glycoprotein M6B (Gpm6B). This glycoprotein is a transmembrane protein related to the proteolipid protein (PLP) family that also includes M6A and the smaller splice variant DM20 [7]. Just recently, the membrane glycoprotein M6A was demonstrated to interact with the $\mu$ opioid receptor, an interaction that facilitates endocytosis and recycling of the receptor [8]. The function of Gpm6B is still largely unknown, although it has been speculated that proteolipid proteins, in addition to their role in structuring of the myelin, might also function as housekeeping proteins involved in intracellular trafficking [9]. Fjorback et al. [10] discovered that coexpression of SERT with Gpm6B mediates a significant decrease in SERT-mediated 5-HT-uptake. In the presence of Gpm6B, the 5-HT uptake was decreased by $50 \%$. Previous studies on 5-HT physiology in mammary gland cells revealed the critical roles of 5-HT in regulating epithelial homeostasis during involution, which is characterized by epithelial tissue regression [11]. One expected effect of elevated 5-HT activity in the normal breast is widespread apoptotic cell death [12].

The second studied TVM with proapoptotic activity was death receptor 6 (DR6). This protein belongs to tumour necrosis factor group (TNF), specifically to death receptor group (DRS: death receptors system). DR6 like other DRS plays role in regulation of cell-specific and inflammatory response of the cell. The primary role is apoptosis induction, but they play role also in cell proliferation, differentiation, and programmed cell death [13]. TNF receptors, in their extracellular part, contain many cysteine residues that are able to bind ligands. After binding ligand to receptor, by the effect of bond and death domain, caspase is activated and apoptosis is initiated. The function of DR6 in apoptosis activation is not clear, because $D R 6$ ligand was not detected yet. The activation of apoptosis by DR6, TRAIL/apoL receptors, or NF-UB, JNK pathways together with TNFRland CD95 is clarified. High expression of DR6 in carcinoma cells that influences antitumour's response by differentiate and proliferative effects on monocytes correlates with high activity of NF-kB [14]. Zeng et al. [15] suggest that DR6-induced apoptosis occurs through a new pathway that is different from the classical pathways mainly through interacting with Bax.

\section{Material and Methods}

2.1. Experimental Design. A total of 110 examined patients were participating in experiment. The experimental group consists of patients $(n=75)$ with breast carcinoma (ductal invasive carcinoma $n=65$; average of age $X=53$, lobular invasive carcinoma $n=8$; average of age $X=57$, and nondifferentiated breast carcinoma $n=2$, average of age $X=44$ ). All patients in the experimental group had a tumour with the size of stage I, but individual grade of tumours was different. The control group consists of 35 women $(n=$ 35 ; average of age $X=51$ ). Women in the control group measured blood pressure and clinical biochemistry results were evaluated along with the overall health status evaluated in standard preventive examinations. Patients and those in the control group responded to the questionnaire. The control group that consisted of people who feel subjectively healthy and preventive examination by the doctor was negative with regard to the current troubles in their health. Screening on comarkers was investigated in the range of reference values for various tumour markers and just had negative sonographic examination of the reproductive organs. Tumour predisposition we take into account with regard to the incidence of cancer among relatives in previous generations. All women in the control group $(n=35)$ were subjected to standard haematological and clinical biochemical laboratory tests (e.g., blood count, coagulation tests, APTT: activated practical thromboplastin time, bleeding time, Quick test, recalcification time, glycemia, lipid profile, total protein, albumin, bilirubin, AST, ALT, ALP, amylase, uric acid, and qualitative urine analysis), which were done by routine haematological and biochemical analytical procedures using bionalyzer Advia, Sysmex Centaur, in cooperation with the complex diagnostic laboratories LABMED in Košice.

2.2. Immunohistochemical Analysis. In all patients from the experimental group ( $n=75)$ histopathological and cytological examination was performed, for confirmation or for establishing the diagnosis. In the laboratory CytoLab Ltd. and the Department of Pathology UNLP in Kosice, health care professionals used the tumour tissue and subjected them to excitation and arrangements necessary for the evaluation of histological slides. Stage and individual grades of tumours were confirmed by histological and immunohistological methods. The basic markers for the determination of breast tumour progression, such as estrogenic receptor clone 1D5, progesterone receptor PgR636, clone D07 of apoptotic protein p53, and Ki67 clone MIB1, were examined by the Department of Laboratory Medicine, University Hospital of L. Pasteur in Košice, using peripheral blood of patients.

2.3. RT-PCR Analysis. To find evidence of changes in mRNA levels, we decided to use RT-PCR. We performed four analyses for each gene per person in experimental and control groups. Blood has been taken from vena mediana cubiti to $\mathrm{K}_{2}$ EDTA covered test tube. RNA was isolated from peripheral blood diagnostic isolation kit (Qiagen). The reverse transcription from mRNA to cDNA was made using 
superscript II (Invitrogen). Normalization of the results was performed by housekeeping gene $\beta$-actin. Amplification of the specific genes $D R 6, G p m 6 B$, and $\beta$-actin ran for 30 cycles $\left(94^{\circ} \mathrm{C} 5 \mathrm{~min}, 94^{\circ} \mathrm{C} 30 \mathrm{~s}, 58.4^{\circ} \mathrm{C} 30 \mathrm{~s}\right.$, and $\left.72^{\circ} \mathrm{C} 45 \mathrm{~s}\right)$, using appropriate primers (Table 1) in the thermocycler 7500 RealTime PCR System (Applied Biosystems). Electrophoresis of individual genes' PCR products was performed on agarose gels. Subsequent quantification was done by measuring fluorescent signal intensity using the GBOX visualization system (Syngene). Numerical quantification of changes in expression levels was evaluated using the DataSyngene program.

2.4. Western Blot Analysis. The proteins from peripheral blood were isolated by using SQ DNA/RNA/protein kit (Omega) and resolved by sodium dodecyl sulphatepolyacrylamide gel electrophoresis (SDS-PAGE). After western blotting (Trans blot SD semidry transfer cell, Biorad) blots on nitrocellulose membrane were probed with a rabbit monoclonal antibody against Gpm6B (Sigma, dilution 1:100) at room temperature for 12 hours and rabbit monoclonal antibody against DR6 (Sigma) at room temperature for 5 hours in dilution 1:1000. For normalization of our data we used $\beta$-actin antibody (Santa Cruz Biotechnology). After being washed by $0.05 \%$ phosphate buffered saline (PBS)-Tween, the membranes were incubated by goat anti-rabbit (Gpm6b, DR6) secondary antibody conjugated by horseradish peroxidase (sc-2033, 1:3000, Santa Cruz Biotechnology) for $1 \mathrm{~h}$ and then washed again by PBS-Tween. Finally, the bands on the membranes were visualized with a SuperSignal West Pico Chemiluminescence Substrate (ECL system from Pierce, \#34080) and detected by GBOX visualization system (Syngene). Spot analysis was made using Gene Tools (SynGene).

2.5. Data Analysis. All experimental groups consisted of $n=$ 110 people. In order to minimize the impact of variability in the experimental people, all samples were measured four times. For the statistical evaluation one-way ANOVA Student-Newmann-Keuls test was used. Data are presented as mean percent \pm SD. Statistical analysis was processed by the program GraphPad INSTAT.

\section{Results and Discussion}

The results of molecular detection of DR6 and Gpm6B expression in patients with breast cancer were compared to control group with no tumour predisposition $(n=35)$. We also analysed 75 patients with breast carcinoma.

In the first step of our work we were primarily analysing levels of serum markers like progesterone receptor clone PgR636, p53 clone D07, Ki67 clone MIB-1, and estrogenic receptor clone 1D5 in the experimental group of patients suffering from histopositive breast carcinoma $(n=75)$. Analysis was not performed in eight patients with invasive lobular breast carcinoma. We detected correlation between increased levels of Ki67 clone MIB-1 and increased level of p53 protein clone D07 during grades progression from GI to GIII of breast carcinoma.

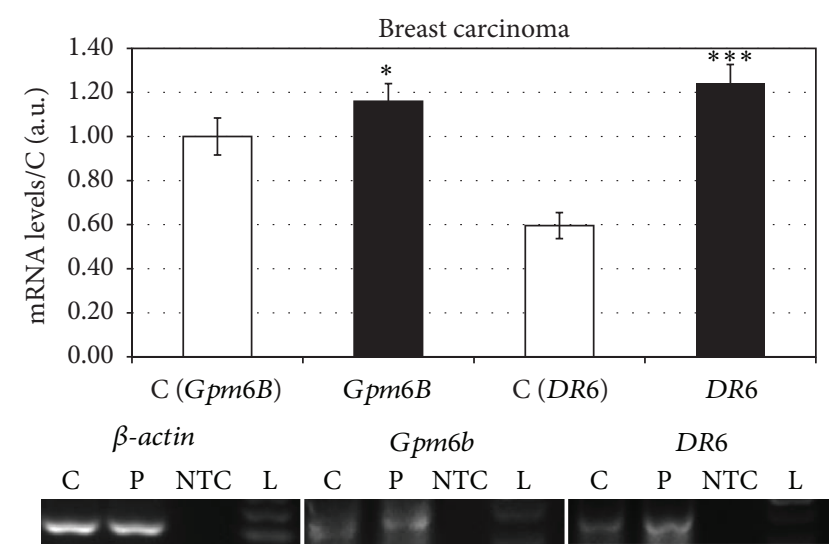

FIGURE 1: Levels of mRNA of DR6 and Gpm6B in breast carcinoma patients.

Both markers are related to worse prognosis and increased metastatic processes. Although the marker Ki-67 is not determined routinely in present, we found that its levels in G1 stage were by $7.5 \%$ higher, in G2 stage were by $19 \%$ higher, and in G3 stage were by $54.9 \%$ higher than controls. Opposite to this we have found correlation between decreased levels of progesterone and estrogenic receptors. Complete results are shown in Table 2.

In the next step of our work we analysed the mRNA expression of DR6, Gpm6B, and $\beta$-actin genes in peripheral blood of patients $(n=75)$ with breast carcinoma (ductal invasive carcinoma $n=65$, lobular invasive carcinoma $n=8$, and nondifferentiated beast carcinoma $n=2$ ). In the group of all patients with ductal invasive carcinoma within all grades, we detected, in comparison to control group, significantly increased $(106 \% \pm 8.3 \%)$ level of DR6 mRNA (Figure 1), that according to Western blot analysis corresponded with increased level (70\% $\pm 8 \%$ ) of protein $\operatorname{Dr} 6$ (Figure 2).

During the detection of $G p m 6 B$ in the same group of patients against control group, we found significantly increased (18 $\pm 3.1 \%$ ) levels of Gpm6B mRNA (Figure 1). This mRNA level was just partially expressed into slightly higher $(20 \pm 5.4 \%)$ level of protein Gpm6B.

The last stage of our research was aimed at finding the correlation between grade of ductal invasive carcinoma (G1 to G3) and expression of DR6 and Gpm6B. In statistical analysis of DR6 mRNA level changes we detected significant increase starting in G1 $(120 \pm 3.2 \%)$ and reached maximal level in G3 (197 $\pm 6.5 \%)$ (Figure 3). This expression on mRNA levels was similar to protein levels, which copy rising tendency of mRNA with maximal value in G3 (80 $\pm 5 \%$ ) (Figure 4).

The results of $G p m 6 B$ were significantly lower. We detected maximal level of mRNA in G3 (40 $\pm 4.2 \%)$ (Figure 5) which corresponded with elevated level of protein Gpm6B in the group of patients with the same grade of tumours $(35 \pm 8.2 \%)$ (Figure 6).

We detected significantly increased $(92 \% \pm 5.5 \%)$ level of DR6 mRNA in patients suffering from invasive lobular carcinoma (Figure 3). The results correlate with protein levels 
TABLE 1: Primer sequences and length of amplified fragments.

\begin{tabular}{lrrr}
\hline Primers/forward/reverse/antibodies & Sequence & Fragment length (bp) & Distributor \\
\hline$\beta$-actin/forward/ & ACACAGGGGAGGTGATAGCAT & $110 \mathrm{bp}$ & Invitrogen \\
$\beta$-actin/reverse/ & ATACATCTCAAGTTGGGGGACAA & $110 \mathrm{bp}$ & Invitrogen \\
$D R 6 /$ forward/ & CCGCCGAGCCACAGCCACGAT & 384 bp & Invitrogen \\
$D R 6 /$ reverse/ & CCCTTTCTTCCGCACACCCCAACC & 384 bp & Invitrogen \\
$G p m 6 B /$ forward/ & TCCTATCACCTGTTCATTGTGG & $171 \mathrm{bp}$ & Invitrogen \\
$G p m 6 B /$ reverse/ & GCAGCAATCTTCCCGACTC & 171 bp & Invitrogen \\
\hline
\end{tabular}

TABLE 2: Results of molecular analysis of breast carcinoma using histochemical detection.

\begin{tabular}{lcccc}
\hline Analytical stage & ER $>1 \%$ clon 1D5 & PR $>1 \%$ clon PgR636 & Ki67\% clon MIB-1 & p53 clon D 07 \\
\hline G 0 & 64 & 46.4 & 11.2 & 33.15 \\
G I & 95 & 86.25 & 19 & 41.5 \\
G II & 81 & 68 & 54.16 & 12 \\
G III & 32,5 & 29.16 & 73.3 \\
\hline
\end{tabular}

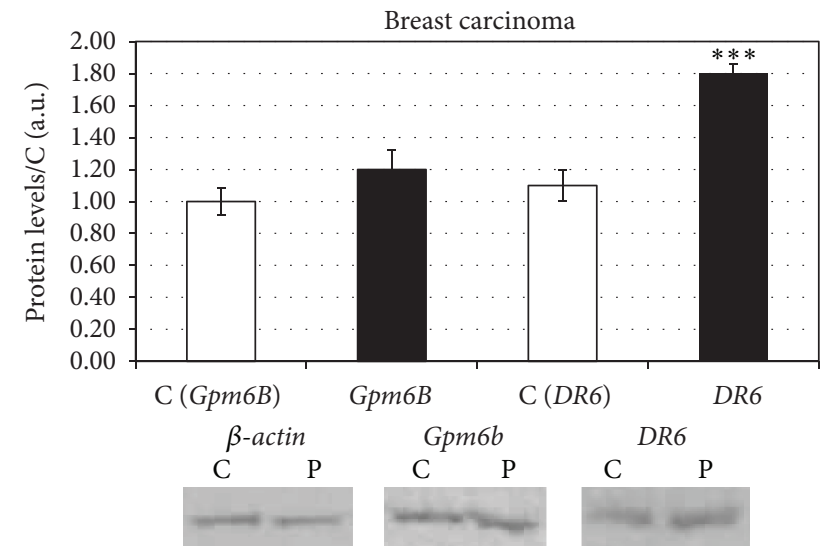

Figure 2: Protein levels of DR6 and Gpm6B in breast carcinoma patients.

of DR6, where we detected significant increase $(40 \% \pm 6 \%)$ (Figure 4).

In the levels of $G p m 6 B$ mRNA from the blood of patients suffering from invasive lobular carcinoma (LIC) we determined increased levels against control group (40 $\pm 5.3 \%)$ (Figure 5).

The levels of protein $G p m 6 B$ were also significantly increased $(22 \% \pm 4.2 \%)$ against control group (Figure 6).

All results of mRNA and protein detection are summarized in Table 3.

Neoangiogenesis is a complicated process, regulated by numerous factors simultaneously and in a coordinated fashion. The process of angiogenesis requires that endothelial cells (ECs) detach from pericytes and the extracellular matrix (ECM), proliferate, migrate, and form capillary tubes which connect to other newly developed vascular tubes. A large number of stimulatory angiogenic factors have been discovered including vascular endothelial growth factor (VEGF), basic fibroblast growth factor (b-FGF), interleukin8, platelet-derived endothelial cell growth factor, tumour necrosis factor alpha, hepatocyte growth factor, angiogenin,

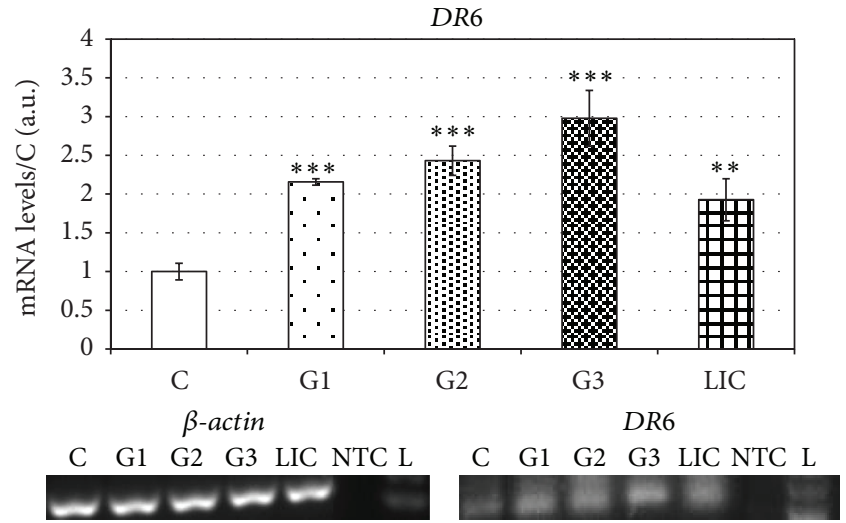

FIgURE 3: Levels of mRNA of DR6 in breast carcinoma patients sorted according to grades.

transforming growth factor alpha, and transforming growth factor beta [16]. This finding suggests that VEGF functions as a survival factor for tumour vessels. VEGF may prevent EC apoptosis through various mediators including Bcl-2, A1, inhibition of apoptosis (IAP), and the PI3-kinase/Akt [17], MAPK/Erk and SAPK/JNK 9 [16], and signal transduction pathways. Gerber et al. [17] observed upregulation of the antiapoptotic protein Bcl-2 and A1 in VEGF treated ECs. Others have shown that the antiapoptotic effects of VEGF in ECs are mediated by activating MAPK/Erk and inhibiting SAPK/JNK signalling pathways [18].

We were monitoring an expression level of proapoptotic DR6 and antiapoptotic Gpm6B isolated from peripheral blood of patients with increased progression of breast cancer, in both mRNA and protein levels. The death ligands and receptors of the TNF/TNFR family have emerged as attractive candidates for modulating tumour cell apoptosis based on their ability to bypass p53-dependent mechanisms in executing programmed cell death [19]. In some cells, called type 1, the DR-initiated extrinsic pathway generates a signal strong enough to initiate apoptosis by itself. However, in the 
TABLE 3: Correlation between grade of breast carcinoma and expression of DR6 and Gpm6B.

\begin{tabular}{lcccc}
\hline $\begin{array}{l}\text { Grade of breast cancer } \\
\text { against controls }\end{array}$ & mRNA levels of $D R 6(\%)$ & $\begin{array}{c}\text { Protein } D R 6 \\
(\%)\end{array}$ & $\begin{array}{c}\text { mRNA levels of } \\
\text { Gpm6B (\%) }\end{array}$ & $\begin{array}{c}\text { Protein Gpm6B } \\
(\%)\end{array}$ \\
\hline Breast carcinoma & $\uparrow 106$ & $\uparrow 70$ & $\uparrow 16$ & $\uparrow 20$ \\
average values & $\uparrow 110$ & $\uparrow 30$ & $\uparrow 6$ & $\uparrow 20$ \\
G I & $\uparrow 143$ & $\uparrow 50$ & $\uparrow 20$ & $\uparrow 40$ \\
G II & $\uparrow 197$ & $\uparrow 80$ & $\uparrow 40$ & $\uparrow 30$ \\
G III & & & \\
\hline
\end{tabular}

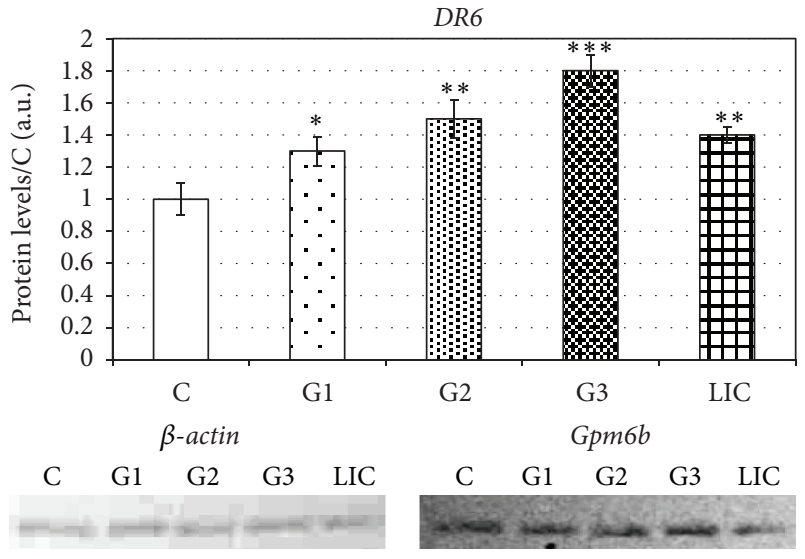

FIGURE 4: Protein levels of DR6 in breast carcinoma patients sorted according to grades.
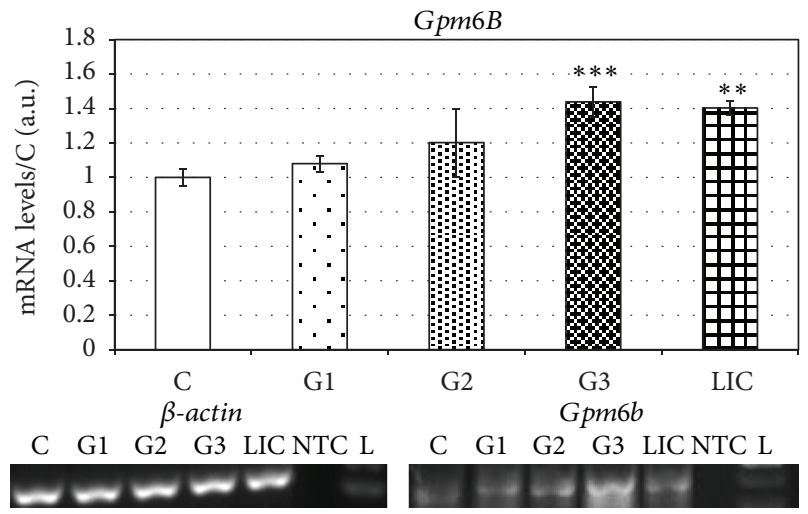

FIGURE 5: Levels of mRNA of Gpm6B in breast carcinoma patients sorted according to grades.

majority of cells, called type 2, the DR-initiated signal needs to be amplified to induce apoptosis. This amplification can be achieved by a cross-talk between the extrinsic pathway and the $\mathrm{Bcl}-2$ regulated mitochondrial, or intrinsic, pathway [20]. One mechanism by which the extrinsic pathway recruits the intrinsic pathway involves caspase- 8 mediated cleavage of the proapoptotic $\mathrm{BH} 3$-only $\mathrm{Bcl}-2$ family member, $\mathrm{Bid}$, to generate active truncated Bid. Active truncated Bid antagonizes the function of prosurvival Bcl-2 family members, such as Bcl2 and Bcl-XL, triggering a sequence of events that culminates in the release of apoptotic factors from the mitochondrion

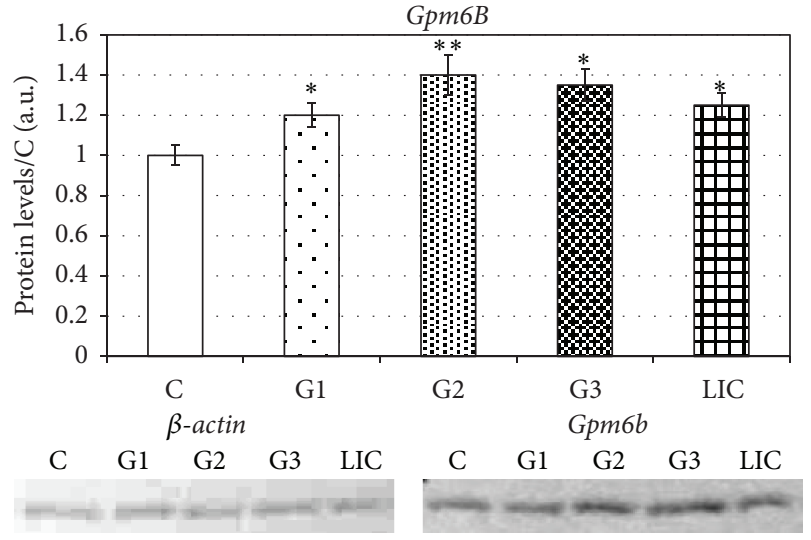

Figure 6: Protein levels of $G p m 6 B$ in breast carcinoma patients sorted according to grades.

[21]. These apoptotic factors mediate activation of caspase9 and antagonize the activity of inhibitors of apoptosis that otherwise function to suppress caspase activity.

Kasof et al. [22] report that the new member of the TNFR family, $D R 6$, induced apoptosis. A primary function for death receptors is to induce apoptosis [23]. Additionally, DR6 may regulate the cytokine-driven differentiation of monocytes to dendritic cells, which suggests, DR6 could play a role in the development of myeloid derived suppressor cells within tumours [14]. DR6 has been reported to be upregulated in numerous solid tumours [20]. DR6 is expressed ubiquitously with high expression in lymphoid organs, heart, brain, and pancreas. Ectopic expression of DR6 in some cell lines leads to apoptosis and activation of the JNK and NF- $\kappa$ B pathways. Some tumour cells overexpress DR6, typically in conjunction with elevated antiapoptosis molecules [14]. DR6 is expressed not only in cancer cells, but also in tumour vascular cells. This expression on host cells in the tumour microenvironment suggests that DR6 may have broad applicability as a tumour biomarker. Recently it was shown that soluble DR6 is the result of matrix metalloproteinase-14 (MMP-14) cleavage of membrane bound DR6 [24]. Thus serum DR6 protein may serve as an indicator of tumour MMP-14 levels. MMP-14 has been correlated with tumour invasion, metastases, and poor patient prognosis [25]. We examined significantly increased mRNA levels of DR6 in all three experimental groups (GIGIII). The mRNA levels of DR6 in patients suffering from breast carcinoma were by $106 \%$ higher than in control group. 
The mRNA levels correlated with the levels of particular proteins. We observed significantly increased (70\%) level of DR6 protein in breast carcinoma. Protein levels were increased in G1 by 30\%, in G2 by $50 \%$, and in G3 by $80 \%$ (Table 3).

These findings correspond with results of Buckanovich et al. [5], who pointed out the increase of DR6 mRNA level by $152 \%$ comparing to healthy patients. Our results as well correlate with Yang et al. [26] who detected overexpression (125\%) of DR6 mRNA in patients suffering from ductal invasive carcinoma.

We were also monitoring the changes in expression of antiapoptotic Gpm6B. Discovery of relationship between coexpressions of SERT and Gpm6B which mediates a significant decrease in SERT-mediated 5-HT-uptake is very important proof of antiapoptotic activity of Gpm6B [10]. 5-HT does not enhance tumour cell proliferation but acts as a regulator of angiogenesis by reducing the expression of matrix metalloproteinase-12 (MMP-12) in tumourinfiltrating macrophages. Tumour-infiltrating macrophages are associated with tumour progression and metastasis. They were reported to regulate angiogenesis in breast (Lin et al.) [27] as well as lung cancer and melanoma (Houghton et al.) [28]. Macrophages are known to specifically secrete MMP-12, which leads to enhanced generation of angiostatin [29] and thereby suppresses tumour growth by halting angiogenesis. We found elevated levels of Gpm6B mRNA in all grades of breast cancer with maximal level in G3. In protein levels we detected maximal level in GII with the constant level of expression in G3. These results showed that antiapoptotic signalling during neovascularization is significantly increased. Building on the results, it will be advisable in the future on study the influence of cytostatic treatment to the expression of genes related to apoptotic pathways and their relationship with progression of breast cancer tumours.

\section{Conclusion}

Formation of new blood vessels and metastasis are two processes that are central to the progression of cancer. As such, they have become important targets for the development of anticancer agents. The paper describes the expression of Dr6 and $G p m 6 B$ as members of apoptotic machinery in correlation with intensity of neovascularization during tumour growth and metastatic processes. We found significant changes of mRNA expression levels in tumour vascular genes DR6 and Gpm6B obtained from peripheral blood of patients suffering from different grades of breast carcinoma. The study also refers to increase of tumour vascular proteins levels coded by DR6 and Gpm6B genes. After confrontation of the results obtained by TVM clinical, biochemical, and histological detection, we found out that increased levels of DR6 proteins in G1 to G3 stages are similar to increased $\mathrm{Ki}$ 67 clone MIB-1. In comparison between DR6 and Gpm6B protein levels it was obvious that protein levels of $D R 6$ were about $60 \%$ higher than $G p m 6 B$ which suggests higher proapoptotic signalization mediated through TNF/TNFR family members.
The study of expression of apoptotic machinery members during neovascularization provides an explanation of the role of angiogenic factors in correlation with breast cancer progression and suggests that this approach could help with the development of effective antiangiogenic therapies useful for treatment of breast cancer.

$\begin{array}{ll}\text { Abbreviations } \\ \text { DRS: } & \text { Death receptors systems } \\ \text { EC: } & \text { Endothelial cell } \\ \text { G1: } & \text { Grade1 } \\ \text { G2: } & \text { Grade2 } \\ \text { G3: } & \text { Grade3 } \\ \text { Gpm6B: } & \text { Glycoprotein M6-b } \\ \text { LIC: } & \text { Lobular invasive carcinoma } \\ \text { NF-kB: } & \text { Nuclear factor } \\ & \text { kappa-light-chain-enhancer of activated } \\ & \text { B cells } \\ \text { NF-UB: } & \text { Ubiquitin signalling in the nf-kappab } \\ & \text { pathway } \\ \text { PgR636: } & \text { Progesterone receptor } \\ \text { PLP: } & \text { Proteolipid protein } \\ \text { SDS-PAGE: } & \text { Sodium dodecyl } \\ & \text { sulphate-polyacrylamide gel } \\ & \text { electrophoresis } \\ \text { TNF: } & \text { Tumour necrosis factor group } \\ \text { TNFR1: } & \text { Tumour necrosis factor receptor } \\ \text { TRAIL: } & \text { TNF-related apoptosis-inducing ligand } \\ \text { TVM: } & \text { Tumour vascular markers } \\ \text { VEGF: } & \text { Vascular endothelial growth factor } \\ \text { VLC: } & \text { Vascular leukocytes. } \\ & \end{array}$

\section{Conflict of Interests}

The authors declare that there is no conflict of interests regarding the publication of this paper.

\section{Acknowledgment}

This research work was supported by Grant Project DIAGONKO ITMS: 26220220153.

\section{References}

[1] S. Sarkar and M. Mandal, "Growth factor receptors and apoptosis regulators: signaling pathways, prognosis, chemosensitivity and treatment outcomes of breast cancer," Breast Cancer, vol. 3, no. 1, pp. 47-60, 2009.

[2] W. Liu, S. A. Ahmad, N. Reinmuth et al., "Endothelial cell survival and apoptosis in the tumor vasculature," Apoptosis, vol. 5, no. 4, pp. 323-328, 2000.

[3] S. Seaman, J. Stevens, M. Y. Yang, D. Logsdon, C. Graff-Cherry, and B. St. Croix, "Genes that distinguish physiological and pathological angiogenesis," Cancer Cell, vol. 11, no. 6, pp. 539554, 2007.

[4] J. R. MacFadyen, O. Haworth, D. Roberston et al., "Endosialin (TEM1, CD248) is a marker of stromal fibroblasts and is not 
selectively expressed on tumour endothelium," FEBS Letters, vol. 579, no. 12, pp. 2569-2575, 2005.

[5] R. J. Buckanovich, D. Sasaroli, A. O’Brien-Jenkins et al., “Tumor vascular proteins as biomarkers in ovarian cancer," Journal of Clinical Oncology, vol. 25, no. 7, pp. 852-861, 2007.

[6] S. W. Fesik, "Promoting apoptosis as a strategy for cancer drug discovery," Nature Reviews Cancer, vol. 5, no. 11, pp. 876-885, 2005.

[7] D. A. Vouyiouklis, H. Werner, I. R. Griffiths, G. J. Stewart, K. Armin-Nave, and C. E. Thomson, "Molecular cloning and transfection studies of M6b-2, a novel splice variant of a member of the PLP-DM20/M6 gene family," Journal of Neuroscience Research, vol. 52, pp. 633-640, 1998.

[8] D.-F. Wu, T. Koch, Y.-J. Liang et al., "Membrane glycoprotein M6a interacts with the $\mu$-opioid receptor and facilitates receptor endocytosis and recycling," Journal of Biological Chemistry, vol. 282, no. 30, pp. 22239-22247, 2007.

[9] N. L. Nadon, S. Miller, K. Draeger, and M. Salvaggio, "Myelin proteolipid DM20: evidence for function independent of myelination," International Journal of Developmental Neuroscience, vol. 15, no. 3, pp. 285-293, 1997.

[10] A. W. Fjorback, H. K. Müller, and O. Wiborg, "Membrane glycoprotein M6B interacts with the human serotonin transporter," Journal of Molecular Neuroscience, vol. 37, no. 3, pp. 191-200, 2009.

[11] V. P. Pai and N. D. Horseman, "Biphasic regulation of mammary epithelial resistance by serotonin through activation of multiple pathways," Journal of Biological Chemistry, vol. 283, no. 45, pp. 30901-30910, 2008.

[12] V. P. Pai, A. M. Marshall, L. L. Hernandez, A. R. Buckley, and N. D. Horseman, "Altered serotonin physiology in human breast cancers favors paradoxical growth and cell survival," Breast Cancer Research, vol. 11, no. 6, article R81, 2009.

[13] M. E. Guicciardi and G. J. Gores, "Life and death by death receptors," The FASEB Journal, vol. 23, no. 6, pp. 1625-1637, 2009.

[14] R. Benschop, T. Wei, and S. Na, "Tumor necrosis factor receptor superfamily member 21: TNFR-related death receptor-6, DR6," Advances in Experimental Medicine and Biology, vol. 647, pp. 186-194, 2009.

[15] L. Zeng, T. Li, D. C. Xu et al., "Death receptor 6 induces apoptosis not through type I or type II pathways, but via a unique mitochondria-dependent pathway by interacting with Bax protein," The Journal of Biological Chemistry, vol. 287, pp. 29125-29133, 2012.

[16] E. Naik, L. A. O’Reilly, M.-L. Asselin-Labat et al., "Destruction of tumor vasculature and abated tumor growth upon VEGF blockade is driven by proapoptotic protein Bim in endothelial cells," Journal of Experimental Medicine, vol. 208, no. 7, pp. 13511358, 2011.

[17] H.-P. Gerber, V. Dixit, and N. Ferrara, "Vascular endothelial growth factor induces expression of the antiapoptotic proteins Bcl-2 and A1 in vascular endothelial cells," Journal of Biological Chemistry, vol. 273, no. 21, pp. 13313-13316, 1998.

[18] K. Gupta, S. Kshirsagar, W. Li et al., "VEGF prevents apoptosis of human microvascular endothelial cells via opposing effects on MAPK/ERK and SAPK/JNK signaling," Experimental Cell Research, vol. 247, no. 2, pp. 495-504, 1999.

[19] L. A. Tartaglia and D. V. Goeddel, “Tumor necrosis factor receptor signaling. A dominant negative mutation suppresses the activation of the $55-\mathrm{kDa}$ tumor necrosis factor receptor," Journal of Biological Chemistry, vol. 267, no. 7, pp. 4304-4307, 1992.
[20] A. Ashkenazi, P. Holland, and S. G. Eckhardt, "Ligand-based targeting of apoptosis in cancer: the potential of recombinant human apoptosis ligand 2/tumor necrosis factor-related apoptosis-inducing ligand (rhApo2L/TRAIL)," Journal of Clinical Oncology, vol. 26, no. 21, pp. 3621-3630, 2008.

[21] R. S. Herbst, R. Kurzrock, D. S. Hong et al., "A first-in-human study of conatumumab in adult patients with advanced solid tumors," Clinical Cancer Research, vol. 16, no. 23, pp. 5883-5891, 2010.

[22] G. M. Kasof, J. J. Lu, D. Liu et al., "Tumor necrosis factor- $\alpha$ induces the expression of DR6, a member of the TNF receptor family, through activation of NF- $\kappa$ B," Oncogene, vol. 20, no. 55, pp. 7965-7975, 2001.

[23] K. Schulze-Osthoff, D. Ferrari, M. Los, S. Wesselborg, and M. E. Peter, "Apoptosis signaling by death receptors," European Journal of Biochemistry, vol. 254, no. 3, pp. 439-459, 1998.

[24] E. M. Tam, C. J. Morrison, Y. I. Wu, M. S. Stack, and C. M. Overall, "Membrane protease proteomics: isotope-coded affinity tag MS identification of undescribed MT1-matrix metalloproteinase substrates," Proceedings of the National Academy of Sciences of the United States of America, vol. 101, no. 18, pp. 6917-6922, 2004.

[25] B. Têtu, J. Brisson, C. S. Wang et al., "The influence of MMP14, TIMP-2 and MMP-2 expression on breast cancer prognosis," Breast Cancer Research, vol. 8, no. 3, article R28, 2006.

[26] K. Yang, C. Mooney, G. Spahlinger et al., "DR6 as a diagnostic and predictive biomarker in adult sarcoma," PLoS ONE, vol. 7, no. 5, Article ID e36525, 2012.

[27] E. Y. Lin, J.-F. Li, L. Gnatovskiy et al., "Macrophages regulate the angiogenic switch in a mouse model of breast cancer," Cancer Research, vol. 66, no. 23, pp. 11238-11246, 2006.

[28] A. M. Houghton, J. L. Grisolano, M. L. Baumann et al., "Macrophage elastase (matrix metalloproteinase-12) suppresses growth of lung metastases," Cancer Research, vol. 66, no. 12, pp. 6149-6155, 2006.

[29] Z. Dong, R. Kumar, X. Yang, and I. J. Fidler, "Macrophagederived metalloelastase is responsible for the generation of angiostatin in Lewis lung carcinoma," Cell, vol. 88, no. 6, pp. 801-810, 1997. 


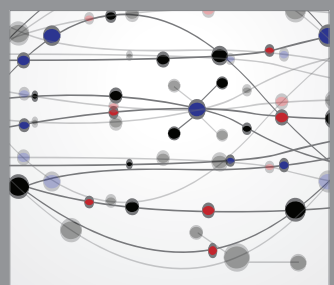

The Scientific World Journal
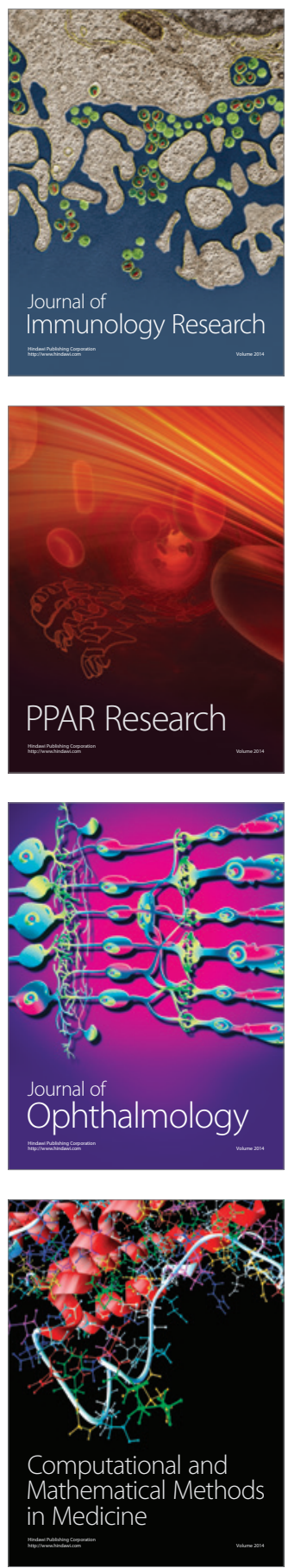

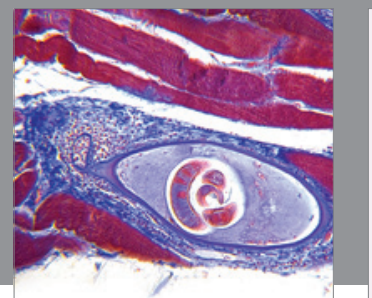

Gastroenterology

Research and Practice
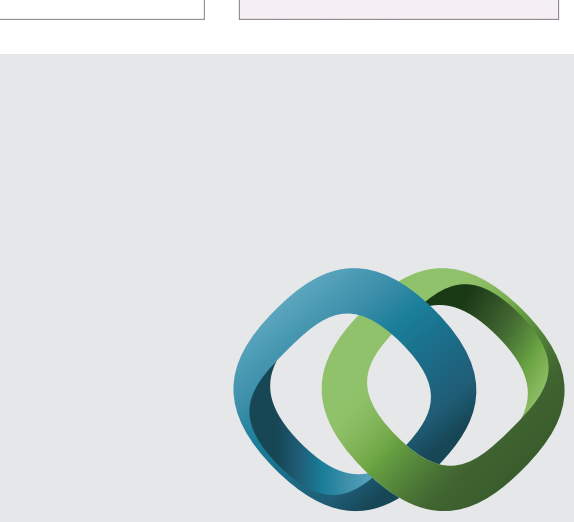

\section{Hindawi}

Submit your manuscripts at

http://www.hindawi.com
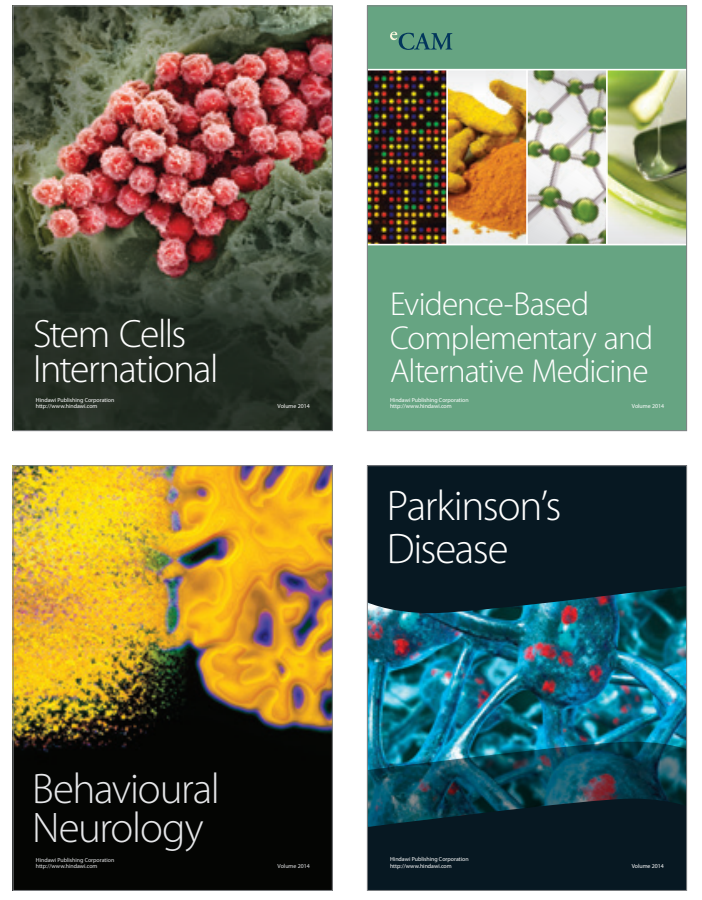
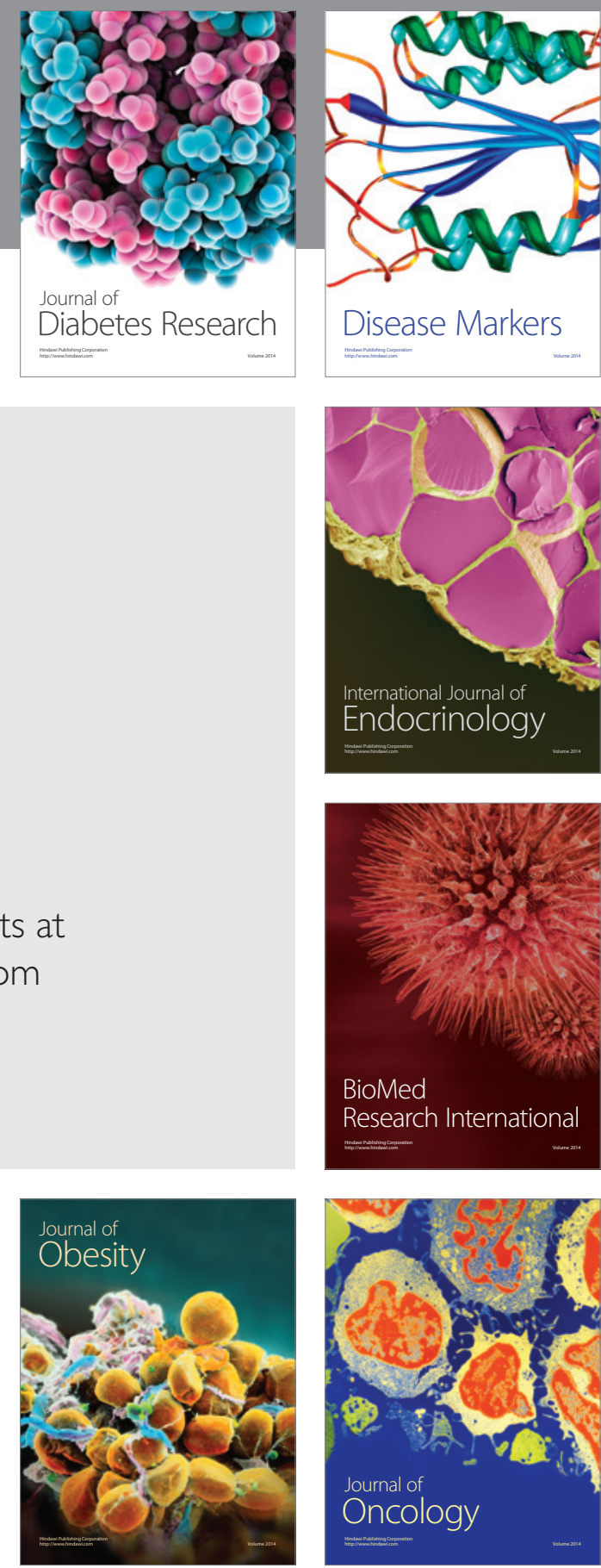

Disease Markers
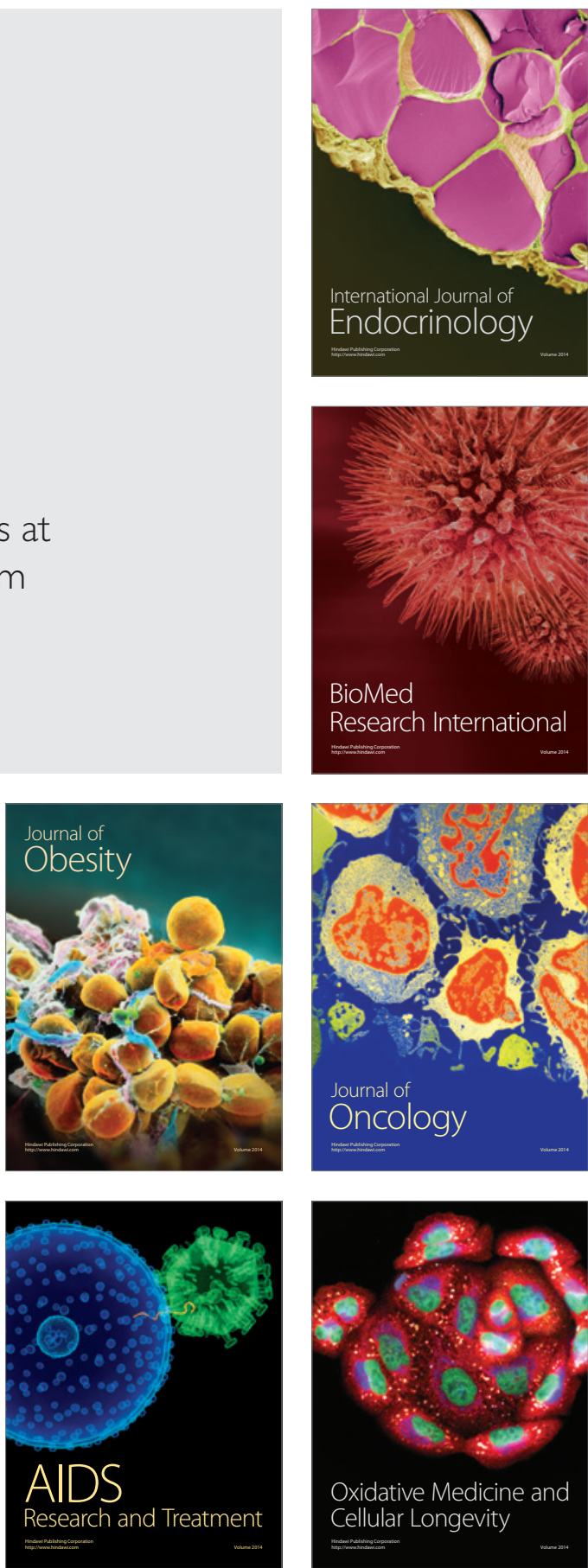